\title{
An Extended Langhaar's Solution for Two-Dimensional Entry Microchannel Flows with High-Order Slip
}

\author{
R. Rasooli and B. Çetin
}

\begin{abstract}
The tremendous advances in micro-fabrication technology have brought numerous applications to the field of micro-scale science and engineering in recent decades. Microchannels are inseparable part of microfluidic technology which necessitate knowledge of flow behavior inside microchannels. For gaseous flows, the mean free path of a gas is comparable with characteristic length of a microchannel due to the micro-scale dimension of the channel. So, no-slip velocity assumption on the boundaries of channel is no longer valid, and a slip velocity needs to be defined. Although rigorous modeling of rarefied flows requires molecular solutions, researchers proposed use of slip models for applicability of the continuum equations. In slip-flow regime (i.e. Knudsen numbers up to 0.1), well-known Maxwell's firstorder slip model is applicable. For higher Knudsen numbers, higher-order slip models can be implemented to extend the applicability limit of the continuum equations. In the present study, Langhaar's assumptions for entrance region of two-dimensional microchannels (microtube, slit-channel and concentric annular microchannel) have been implemented using high-order slip models. Different slip models proposed in the literature have been used and velocity profile, entrance length and apparent friction factor have been obtained in integral forms.
\end{abstract}

Keywords Microchannel flow $\cdot$ High-order slip · Langhaar's solution

\section{Introduction}

In recent decades, progresses in micro-fabrication technology have open up countless opportunities for many applications as an extension of theoretical micro-scale science by enabling fabrication of micro-scale devices using photolithographic [1], high precision mechanical machining $[1,2]$ and laser machining methods $[3,4]$. Through

R. Rasooli • B. Çetin ( $\square)$

Microfluidics and Lab-on-a-chip Research Group, Mechanical Engineering Department,

İ.D. Bilkent University, 06800 Ankara, Turkey

e-mail: barbaros.cetin@bilkent.edu.tr 
these progresses, even fabrication of structures composed of tubes with diameters of nanometers have become possible [5]. Microchannels and microtubes are inseparable part of microfluidic devices, and play a crucial role which necessitates fundamental knowledge of flow behavior at micro-scale for effective and optimal design of these devices. Many theoretical investigations have been made on the physics of fluid flow in channels and ducts at micro-scale [6-8].

One important effect at micro-scale is rarefaction. As characteristic length $(L)$ of flow approaches to the mean-free-path $(\lambda)$ of the fluid, continuum approach fails to be valid and fluid flow modeling moves from continuum to molecular modeling. Rarefaction is characterized by Knudsen number $(\mathcal{K} n=\lambda / L)$. Continuum hypothesis applies for $\mathcal{K}_{n} \lesssim 10^{-3}$. For $10^{-1} \lesssim \mathcal{K}_{n} \lesssim 10$, the regime is known as transition regime in which continuum equations fail to model the fluid flow. In this regime, molecular models such as DSMC and MD, or solutions of Boltzmann Transport Equation is required. The regime where $10^{-3} \lesssim \mathcal{K} n \lesssim 10^{-1}$ is slip-flow regime in which continuum equations need to be modified through velocity-slip and temperature-jump boundary conditions to take into account molecular interactions of fluid particles with the solid bodies within the Knudsen layer [6].

Following a non-dimensionalization with a reference length and velocity scale, the general form of a velocity-slip boundary condition for an isothermal flow can be written as [8-11]:

$$
U_{s}-U_{\text {wall }}=A_{1} \mathcal{K} n\left(\partial_{n} U\right)_{\text {wall }}+A_{2} \mathcal{K} n^{2}\left(\partial_{n}^{2} U\right)_{\text {wall }},
$$

where $\left(\partial_{n}\right)$ is gradient in the normal direction of the solid boundary, $A_{1}$ and $A_{2}$ are the slip-coefficients. Many researchers proposed to employ different coefficients based on kinetic theory of rarefied gases [9, p. 74]. For a fully-diffuse reflection, proposed coefficients in the literature are tabulated in Table 1 . The first term is called as Maxwell's first-order slip, and inclusion of the second term turns the boundary condition into a second-order one. Approximation of Boltzmann Transport equation up to $\mathcal{O}(\mathcal{K} n)$ (i.e. first order in $\mathcal{K} n$ ) results in compressible form of Navier-Stokes equations which require only the first-order boundary conditions. Approximations with higher order terms result in higher order equations such as Burnett and Woods equations. However, researchers showed that application of the second-order boundary condition with Navier-Stokes equations [7, 12-15] or first-order Maxwell boundary

Table 1 Coefficients for different slip models

\begin{tabular}{l|l|l}
\hline Model & $\mathbf{A}_{\mathbf{1}}$ & $\mathbf{A}_{\mathbf{2}}$ \\
\hline First-order Model & 1.0 & 0 \\
\hline Schamberg Model & 1.0 & $-5 \pi / 12$ \\
\hline Cercignani Model & 1.1466 & -0.9756 \\
\hline Deissler Model & 1.0 & $-9 / 8$ \\
\hline Hsia Model & 1.0 & $-1 / 2$ \\
\hline Mitsuya Model & 1.0 & $-2 / 9$ \\
\hline
\end{tabular}


condition with second-order quasi-hydyrodynamic equations [7] may extend applicability of the slip-flow regime up to $\mathcal{K} n \approx 0.25$. The second-order term in the boundary condition may introduce some numerical difficulties associated with accurate calculation of the high-order derivatives, especially for complex geometries [9]. Therefore, some studies introduced higher-order accurate boundary conditions which include only first-order derivatives [16, 17]. Considering diffuse reflections of gas molecules, Beskok and Karniadakis [16] proposed a general velocity-slip boundary condition (will be referred as Beskok Model hereafter) as:

$$
U_{s}-U_{\text {wall }}=\frac{\mathcal{K} n}{1-b \mathcal{K} n}\left(\partial_{n} U\right)_{\text {wall }}
$$

where $b$ is a general slip coefficient. Strictly speaking, parameter $b$ is a function of $\mathcal{K} n$. For a general choice, Eq. (2) is first-order in $\mathcal{K} n$. However, for a specific choice of the parameter $b$, the boundary condition can be transformed into a second-order one. For slip-flow regime, Beskok and Karniadakis [16] derived a condition to ensure second-order accuracy of (2) as:

$$
b=\left(\frac{1}{2} \frac{\partial_{n}^{2} U_{o}}{\partial_{n} U_{o}}\right)_{\text {wall }},
$$

where $U_{o}$ is the velocity-profile corresponding to a no-slip case (i.e. $\mathcal{K} n=0$ ).

For pressure-driven flows in microchannels, on top of rarefaction effect, lowMach-number compressibility effect comes into picture due to high viscous resistance leading to a nonlinear streamwise pressure variation [18-20]. That is to say, the fluid flow requires compressible modeling to be able to observe this non-linear streamwise pressure. However, considering short microchannels and/or microchannel flow with low inlet/exit pressure ratio, compressibility effect may be negligible, and the fluid flow can be modeled as incompressible. Moreover, length-over-diameter ratio is typically large for microchannels, which implies entrance effects are negligible. However, the entrance effects may become quite significant for microchannels and nanotubes with small length-over-diameter ratio and micropores as a part of larger scale porous structures.

Study of the entrance effects requires solution of a hydrodynamic entrance problem. The hydrodynamic entrance problem for an incompressible fluid flow was well studied for macroscale several decades ago [21-24]. Same problem has been recently re-visited for microscale [8, 13, 25, 26] for different geometries. Although the hydrodynamic entrance problem is not a true boundary layer problem, boundary layer idealizations, which neglect axial diffusion of momentum and radial pressure gradient, are reasonable approximations for laminar flow problems in ducts [21, 22, 26]. Strictly speaking, this type of idealization leads to a solutions independent of Reynolds number $(R e)$ and suitable for high $R e$ flows. To see the whole picture, full Navier-Stokes equations with presence of axial momentum diffusion and radial pressure gradient need to be solved for hydrodynamic entrance (i.e. entry flow) problems. Solution of full Navier-Stokes equations in the entrance region leads to a 
peculiar behavior. Velocity overshoots with two symmetric velocity maxima off the centerline was observed both in numerical [25, 27] and experimental [28] studies. Main reason of this velocity overshoot is result of the sudden velocity change at the inlet of a channel due to the no-slip boundary condition. The velocity overshoot is significant especially for strictly uniform velocity inlet which is actually an idealization for practical applications. It was observed that the velocity overshoot is much weaker for irrotational inlet velocity profiles, or for inlet velocity profiles which are approximated to a uniform one. The overshoot is only significant at the vicinity of the channel entrance. The zone where this overshoot can be observed is a function of $R e$. Therefore, if measurement data is not gathered close to the inlet of a channel, the velocity overshoot cannot be detected experimentally [29]. One would expect a weaker velocity overshoot for the flows in a microchannel, since sudden change of the velocity at the inlet due to no-slip boundary condition also reduces with the presence of slip-velocity at the channel wall [25].

At macroscale, following boundary layer idealizations, analytical solutions were derived employing different methods such as matching method (based on perturbation analysis), integral method, linearization methods [30, pp. 68-73]. Matching and integral method results in discontinuous solutions for the velocity gradients and pressure distributions. Alternatively, linearization methods yield continuous solutions, but transverse velocity components may not be predicted rigorously. Three different linearization procedures are possible: (i) Langhaar's linearization [21], (ii) Targ's linearization [30] and (iii) Sparrow's linearization [22]. The latter two require solution of an eigenvalue problem which may have some convergence issues especially for regions close to the inlet. Langhaar's linearization, on the other hand, offers an integral type solution with a satisfactory solution at the centerline, in the vicinity of the inlet and far downstream. At macroscale, Langhaar's linerization was implemented for the different geometries such as tube [21], annular tube [31], parallel plate and rectangular ducts $[30,32]$.

Many researchers devoted great effort on experimental investigations and proposed different techniques and experimental protocols [18, 19, 33, 34], but still measurement of different flow parameters to understand fluid physics is a challenging task. At this point, analytical and numerical models serve as a basis for fundamental understanding of the phenomena despite the fact that they require some empirical input parameters in the form of slip-coefficients. In addition, analytical and numerical models serve as a fast way to investigate different scenarios. Present note reports an analytical solution of a 2D incompressible, isothermal flow in a developing region of a microchannel considered both in cylindrical and cartesian coordinates (see Fig. 1) based on Langhaar's linearization. For rarefaction effect, different second-order models are included in the analysis. Moreover, the general velocity-slip boundary condition proposed by Beskok and Karniadakis [16] for which the general slip-coefficient is evaluated with high accuracy based on a no-slip case, is implemented. Although second-order methods extend the applicability of the continuum equations, theoretical background of the models is still a debate [14, 15], and an active research field. The current model is presented with second-order models available in the literature. In the light of new studies, the coefficients of the slip models may be revised 


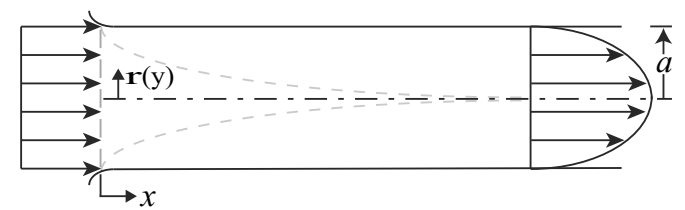

(a) Microchannel in cylindrical coordinates (microtube with a radial coordinate $r$ ) and cartesian coordinates (slit-channel with a transverse coordinate $y$ )
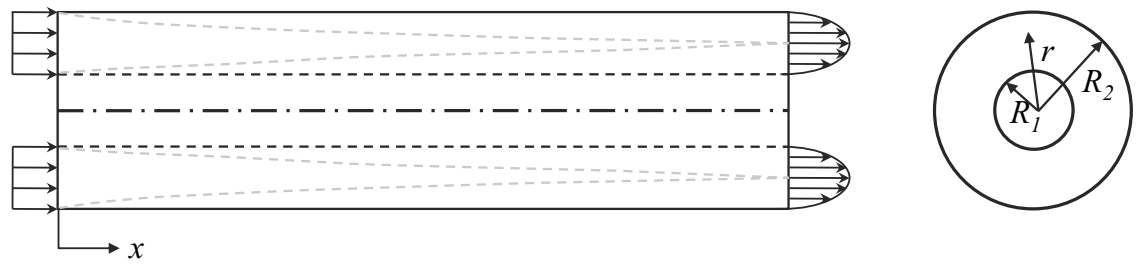

(b) Concentric annular microchannel

Fig. 1 Schematics of the 2-dimensional microchannel problems

$[15,35]$ and so does the current analytical model. By no means, the authors would like to question the validity of the higher order models considered, rather the extension of Langhaar's solution including higher order terms is presented in this note to obtain integral form of the developing velocity profiles inside microchannels. Furthermore, hydrodynamic entrance length and apparent friction factor which are quantities of interest for many engineering calculations are also presented based on the results derived with lineraization. Analytical nature of the model enables a fast evaluation of the velocity field without any need for calculation of eigenvalues. Furthermore, it also enables implementation of Beskok general slip-model with high accuracy. The proposed model may be extended for the exploration of heat transfer problems at the incompressible limit (which has been studied in the literature [36-39] for microchannels in the combined entrance region without any need for numerical calculation of the flow field.

\section{Mathematical Modeling}

\subsection{Velocity Profile}

Assuming an incompressible fluid with constant properties, following Prandtl boundary layer idealizations, the equation of motion in the axial direction ( $x$ being the axial direction) together with continuity equation can be written as:

$$
\nabla \cdot \mathbf{u}=0
$$




$$
\mathbf{u} \cdot \nabla u_{x}=\frac{1}{\rho} \partial_{x} p+\nu \nabla^{2} u_{x}
$$

where $\mathbf{u}$ is the velocity field, $p$ is the pressure field, $u_{x}$ is the velocity component in the axial direction, $\rho$ is the density and $\nu$ is the dynamic viscosity. Moreover, following boundary layer idealization, (i) the diffusive term in the axial direction can be neglected compared to the components in the transverse direction, (ii) the transverse velocities within the channel can be assumed to be small compared to the axial velocity, and (iii) the pressure gradient in the axial direction can be taken as a function of axial coordinate only [21, 32].

\subsubsection{Microtube and Slit-Channel}

With the following dimensionless parameters

$$
\lambda=\frac{u_{x}}{U_{m}}, \eta=\frac{u_{l}}{U_{m}}, q=\frac{r}{a}, \sigma=\frac{x}{R e_{D_{h}} \cdot D_{h}}, \tilde{p}=\frac{p}{1 / 2 \rho U_{m}^{2}},
$$

where $U_{m}$ is the mean velocity (which is also the uniform inlet velocity), $u_{l}$ is the velocity in the transverse direction, $a$ is the scale for the channel height ( $a=R$ for a microtube and half of the channel height for a slit-channel) and $R e_{D_{h}}$ is the $R e$ based on hydraulic diameter, Eqs. (4) and (5) can be non-dimensionalized as:

$$
\begin{gathered}
2^{k-2} \frac{\partial \lambda}{\partial \sigma}+\frac{\operatorname{Re}_{D_{h}}}{q^{k}} \frac{\partial\left(\eta q^{k}\right)}{\partial q}=0 \\
4^{k-2} \lambda \frac{\partial \lambda}{\partial \sigma}+2^{k-2} \operatorname{Re}_{D_{h}} \eta \frac{\partial \lambda}{\partial q}=-2^{2 k-5} \frac{\partial \tilde{p}}{\partial \sigma}+\frac{1}{q^{k}} \frac{\partial}{\partial q}\left(q^{k} \frac{\partial \lambda}{\partial q}\right),
\end{gathered}
$$

where $k=0$ corresponds to flow in a slit-channel (i.e. 2D problem in cartesian coordinates) and $k=1$ corresponds to the flow in a microtube (i.e. $2 \mathrm{D}$ problem in cylindrical coordinates). Parameter $k$ is defined to derive the velocity profile on a common ground for both coordinate systems.

Following Langhaar's linearization [21, 32], the convective terms on the right hand side can be replaced by $\left(\gamma^{2} \lambda\right)$, and Eq. (8) can be written as:

$$
\frac{1}{q^{k}} \frac{\partial}{\partial q}\left(q^{k} \frac{\partial \lambda}{\partial q}\right)-\gamma^{2} \lambda=2^{2 k-5} \frac{\partial \tilde{p}}{\partial \sigma} .
$$

The solution can be expressed as a combination of modified Bessel's and hyperbolic functions as:

$$
\lambda(\gamma, q)=\mathcal{A}(\gamma) \mathcal{G}_{1}(\gamma q)+\mathcal{B}(\gamma) \mathcal{G}_{2}(\gamma q)+\mathcal{C}(\gamma)
$$


where

$$
\begin{aligned}
\mathcal{G}_{1}(\gamma q) & =k \mathrm{I}_{0}(\gamma q)+(1-k) \cosh (\gamma q) \\
\mathcal{G}_{2}(\gamma q) & =k \mathrm{I}_{1}(\gamma q)+(1-k) \sinh (\gamma q) \\
\mathcal{C}(\gamma) & =-\frac{2^{2 k-5}}{\gamma^{2}} \frac{\partial \tilde{p}}{\partial \sigma}
\end{aligned}
$$

Coefficients $\mathcal{A}(\gamma), \mathcal{B}(\gamma)$ and $\mathcal{C}(\gamma)$ can be obtained using the boundary condition at the channel wall:

$$
\begin{gathered}
\lambda^{(s)}(\gamma)=-\left.2^{2-k} A_{1} \mathcal{K} n \frac{\partial \lambda}{\partial q}\right|_{q=1}+\left.4^{2-k} A_{2} \mathcal{K} n^{2} \frac{\partial^{2} \lambda}{\partial q^{2}}\right|_{q=1} \\
\lambda^{(g)}(\gamma)=-\left.2^{2-k} \frac{\mathcal{K} n}{1-b \mathcal{K} n} \frac{\partial \lambda}{\partial q}\right|_{q=1}
\end{gathered}
$$

where the superscript $(s)$ stands for the second-order slip in generic form, and $(g)$ stands for the general-slip model (i.e. Beskok Model). The symmetry boundary condition at the centerline together with the continuity equation reads as:

$$
\int_{0}^{1} q^{k} \lambda d q=2^{-k}
$$

Note that, $b$ is a function of $(\sigma)$ in the entrance region, and reaches a value of -2.0 at the region far from the entrance (i.e. fully-developed region).

The velocity profile can be obtained as:

$$
\lambda(\gamma, q)=\frac{\alpha_{1} \gamma^{n} \mathcal{G}_{1}(\gamma)+\alpha_{2} \mathcal{G}_{2}(\gamma)-\gamma \alpha_{1}^{n} \mathcal{G}_{1}(\gamma q)}{\alpha_{1} \gamma^{n} \mathcal{G}_{1}(\gamma)+\left(\alpha_{2}-2^{k} \alpha_{1}^{n}\right) \mathcal{G}_{2}(\gamma)}
$$

where

$$
\begin{aligned}
& \alpha_{1}=(1-n) \gamma\left(1-4^{2-k} A_{2} \gamma^{2} \mathcal{K} n^{2}\right)+n(1-b \mathcal{K} n), \\
& \alpha_{2}=(1-n) \gamma^{2}\left(2^{2-k} A_{1} \mathcal{K} n+k 4^{k} A_{2} \mathcal{K} n^{2}\right)+2^{2-k} n \gamma^{2} \mathcal{K} n .
\end{aligned}
$$

$n=0$ corresponds to a second-order slip, and $n=1$ corresponds to a general-slip model.

The coefficient $b$ can be derived using Eq. (3) as:

$$
b=-2 \gamma \frac{\mathcal{G}_{1}(\gamma)}{\mathcal{G}_{2}(\gamma)}+2 k
$$

To complete the solution, the relation between the parameters $\gamma$ and $\sigma$ needs to be determined. For this purpose, Eq. (8) can be integrated over the cross-section as: 


$$
\begin{aligned}
\int_{0}^{1}\left(4^{k-2} \lambda \frac{\partial \lambda}{\partial \sigma}+2^{k-2} \operatorname{Re}_{D_{h}} \eta \frac{\partial \lambda}{\partial q}\right) q^{k} d q= & -\int_{0}^{1}\left(2^{2 k-5} \frac{\partial \tilde{p}}{\partial \sigma}\right) q^{k} d q \\
& +\int_{0}^{1} \frac{1}{q^{k}} \frac{\partial}{\partial q}\left(q^{k} \frac{\partial \lambda}{\partial q}\right) q^{k} d q
\end{aligned}
$$

The first integral can be simplified using continuity equation, Eq. (7), as:

$$
\int_{0}^{1}\left(4^{k-2} \lambda \frac{\partial \lambda}{\partial \sigma}+2^{k-2} \operatorname{Re}_{D_{h}} \eta \frac{\partial \lambda}{\partial q}\right) q^{k} d q=4^{k-2} \frac{d}{d \sigma} \int_{0}^{1} \lambda^{2} q^{k} d q
$$

The second integral can be simplified by writing equation of motion for the central core of the channel:

$$
-\int_{0}^{1}\left(2^{2 k-5} \frac{\partial \tilde{p}}{\partial \sigma}\right) q^{k} d q=\frac{2^{2 k-5}}{k+1} \frac{d \lambda_{0}^{2}}{d \sigma}-\left.\frac{\partial^{2} \lambda}{\partial q^{2}}\right|_{q=0},
$$

where the subscript ("0") refers to the values at the centerline. Introducing two new functions $\mathrm{F}$ and $\mathrm{G}$ as:

$$
\begin{aligned}
& F(\gamma)=2 \int_{0}^{1} \lambda^{2} q^{k} d q-\frac{\lambda_{0}^{2}}{k+1} \\
& G(\gamma)=2^{5-2 k}\left(\left.\frac{\partial \lambda}{\partial q}\right|_{q=1}-\left.\frac{\partial^{2} \lambda}{\partial q^{2}}\right|_{q=0}\right)
\end{aligned}
$$

Equation (18) can be written as:

$$
\frac{d \sigma}{d \gamma}=\frac{F^{\prime}(\gamma)}{G(\gamma)}
$$

Functions $F(\gamma)$ and $G(\gamma)$ are written in closed form as:

$$
\begin{gathered}
F(\gamma)=\frac{2}{\mathcal{H}^{2}}\left[\frac{\mathcal{S}^{2}}{k+1}-2 \mathcal{S} \alpha_{1}^{n} \mathcal{G}_{2}+\frac{1}{2} \gamma^{2} \alpha_{1}^{2 n}\left[\mathcal{G}_{1}^{2}-\mathcal{G}_{2}^{2}+\frac{1-k}{\gamma} \mathcal{G}_{1} \mathcal{G}_{2}\right)\right] \\
-\frac{1}{k+1}\left(\frac{\mathcal{S}-\gamma \alpha_{1}^{n}}{\mathcal{H}}\right)^{2} \\
G(\gamma)=2^{5-2 k} \frac{\gamma^{2} \alpha_{1}^{n}\left(\gamma-\mathcal{G}_{2}\right)}{\mathcal{H}}
\end{gathered}
$$

where

$$
\begin{aligned}
\mathcal{S}(\gamma) & =\gamma^{n} \alpha_{1} \mathcal{G}_{1}+\alpha_{2} \mathcal{G}_{2} \\
\mathcal{H}(\gamma) & =\mathcal{S}-2^{k} \alpha_{1}^{n} \mathcal{G}_{2}
\end{aligned}
$$


By integrating Eq. (22), the relation between $\sigma$ and $\gamma$ can be defined as:

$$
\sigma=-\int_{\gamma}^{\infty} \frac{F^{\prime}(\tilde{\gamma})}{G(\tilde{\gamma})} d \tilde{\gamma}
$$

Once the $\gamma$ value is selected, Eqs. (15) and (26) defines the velocity profile within the microchannel.

Using Eqs. (7) and (22), the lateral velocity can be obtained as:

$$
\eta=-\frac{1}{4 \operatorname{Re}_{D_{h}}} \frac{G(\gamma)}{F^{\prime}(\gamma)} \frac{\partial}{\partial \gamma} \mathcal{Q}(\gamma, q)
$$

where the function $\mathcal{Q}(\gamma, q)$ is expressed as:

$$
\mathcal{Q}(\gamma, q)=\frac{q \mathcal{S}(\gamma)-2^{k} \alpha_{1}^{n} \mathcal{G}_{2}(\gamma q)}{\mathcal{H}(\gamma)}
$$

\subsubsection{Concentric Annular Microchannel}

With the following dimensionless parameters

$$
\lambda=\frac{u_{x}}{U_{m}}, \eta=\frac{u_{l}}{U_{m}}, q=\frac{r}{R_{2}}, \sigma=\frac{x}{R e \cdot R_{2}}, \tilde{p}=\frac{p}{1 / 2 \rho U_{m}^{2}},
$$

where $U_{m}$ is the mean velocity (which is also the uniform inlet velocity), $u_{l}$ is the velocity in the transverse direction, $R_{1}$ is the inner radius, $R_{2}$ is the outer radius and $R e$ is the Reynolds number based on the outer radius of channel, Eqs. (4) and (5) can be non-dimensionalized for a concentric annular microchannel as:

$$
\begin{gathered}
\frac{\partial \lambda}{\partial \sigma}+\frac{\operatorname{Re}}{q} \frac{\partial(\eta q)}{\partial q}=0 \\
\lambda \frac{\partial \lambda}{\partial \sigma}+\operatorname{Re} \eta \frac{\partial \lambda}{\partial q}=-\frac{1}{2} \frac{\partial \tilde{p}}{\partial \sigma}+\frac{1}{q} \frac{\partial}{\partial q}\left(q \frac{\partial \lambda}{\partial q}\right)
\end{gathered}
$$

Similarly, by employing Langhaar's linearization method [21, 32], the left hand side term (convective term) can be replaced by $\left(\gamma^{2} \lambda\right)$, and Eq. (31) reads as:

$$
\frac{1}{q} \frac{\partial}{\partial q}\left(q \frac{\partial \lambda}{\partial q}\right)-\gamma^{2} \lambda=\frac{1}{2} \frac{\partial \tilde{p}}{\partial \sigma}
$$

The solution is obtained as a combination of modified Bessel's functions as:

$$
\lambda(\gamma, q)=\mathcal{A}(\gamma) I_{0}(\gamma q)+\mathcal{B}(\gamma) K_{0}(\gamma q)+\mathcal{C}(\gamma)
$$


Coefficients $\mathcal{A}(\gamma), \mathcal{B}(\gamma)$ and $\mathcal{C}(\gamma)$ can be found using the boundary condition at the channel wall,

$$
\begin{gathered}
\lambda^{(f)}(q=1)=-2 \mathcal{K} n\left(\frac{\partial \lambda}{\partial q}\right)_{q=1}, \quad \lambda^{(f)}(q=m)=2 \mathcal{K} n\left(\frac{\partial \lambda}{\partial q}\right)_{q=m} \\
\lambda^{(g)}(q=1)=-2 \frac{\mathcal{K} n}{1-b_{1} \mathcal{K} n}\left(\frac{\partial \lambda}{\partial q}\right)_{q=1}, \quad \lambda^{(g)}(q=m) \quad=2 \frac{\mathcal{K} n}{1-b_{2} \mathcal{K} n}\left(\frac{\partial \lambda}{\partial q}\right)_{q=m}
\end{gathered}
$$

and the continuity equation reads as:

$$
\int_{m}^{1} q \lambda d q=\frac{1}{2}\left(1-m^{2}\right)
$$

where $m$ is the radii ratio of the channel $\left(m=R_{1} / R_{2}\right)$. Note that only the first-order and Beskok model is considered for the concentric tube without loss of generality of the modeling. Velocity profile can be obtained using Eqs. (33)-(36). To discuss the second-order effectsClosed form solution of the velocity profile can be found in the Appendix. The coefficient $b$ can be written both for the inner and outer walls as:

$$
b_{1}=2\left(\frac{u_{x, 0}^{\prime \prime}}{u_{x, 0}^{\prime}}\right)_{q=m}, \quad b_{2}=-2\left(\frac{u_{x, 0}^{\prime \prime}}{u_{x, 0}^{\prime}}\right)_{q=1}
$$

and can be written in closed form as:

$$
\begin{aligned}
& b_{1}=\frac{2 \gamma^{2} m\left[K_{0}(\gamma) I_{0}(\gamma m)-I_{0}(\gamma) K_{0}(\gamma m)\right]}{\gamma m\left[K_{0}(\gamma) I_{1}(\gamma m)+I_{0}(\gamma) K_{1}(\gamma m)\right]-1}-\frac{2}{m} \\
& b_{2}=2\left\{\frac{\gamma^{2}\left[K_{0}(\gamma) I_{0}(\gamma m)-I_{0}(\gamma) K_{0}(\gamma m)\right]}{\gamma I_{1}(\gamma) K_{0}(\gamma m)+\gamma K_{1}(\gamma) I_{0}(\gamma m)-1}+1\right\}
\end{aligned}
$$

Furthermore, the asymptotic values of $b_{1}$ and $b_{2}$ in far distance downstream (i.e. fully-developed region) can be obtained as:

$$
\begin{aligned}
b_{1}^{(f d)} & =2 \frac{m^{2}(1+2 \log m)-1}{m\left[1+m^{2}(2 \log m-1)\right]} \\
b_{2}^{(f d)} & =2 \frac{m^{2}+2 \log m-1}{m^{2}-(1+2 \log m)}
\end{aligned}
$$

To obtain the relation between parameters $\sigma$ and $\gamma$, the axial momentum equation, Eq. (31), should be integrated over the cross-section of the channel as:

$$
\int_{m}^{1}\left(\lambda \frac{\partial \lambda}{\partial \sigma}+\operatorname{Re} \eta \frac{\partial \lambda}{\partial q}\right) q d q=\int_{m}^{1}\left(-\frac{1}{2} \frac{\partial \tilde{p}}{\partial \sigma}\right) q d q+\int_{m}^{1} \frac{1}{q} \frac{\partial}{\partial q}\left(q \frac{\partial \lambda}{\partial q}\right) q d q
$$


The first integral can be simplified using the continuity equation as:

$$
\int_{m}^{1}\left(\lambda \frac{\partial \lambda}{\partial \sigma}+\operatorname{Re} \eta \frac{\partial \lambda}{\partial q}\right) q d q=\frac{d}{d \sigma} \int_{m}^{1} \lambda^{2} q d q
$$

Assuming the central core of the velocity is not affected by viscous effects, the first integral at the right hand side can be simplified as:

$$
\int_{m}^{1}\left(-\frac{1}{2} \frac{\partial \tilde{p}}{\partial \sigma}\right) q d q=\frac{1}{2} \frac{d}{d \sigma} \int_{m}^{1} \bar{\lambda}^{2} q d q-\frac{1-m^{2}}{2}\left(\frac{\partial^{2} \lambda}{\partial q^{2}}\right)_{q=\frac{m+1}{2}}
$$

where the bar values refer to the values at the mean radius of the pipe. The last integral can also be readily evaluated as:

$$
\int_{m}^{1} \frac{1}{q} \frac{\partial}{\partial q}\left(q \frac{\partial \lambda}{\partial q}\right) q d q=\left(\frac{\partial \lambda}{\partial q}\right)_{q=1}-m\left(\frac{\partial \lambda}{\partial q}\right)_{q=m}
$$

Substituting all these equations in the Eq. (40) and re-arranging results in:

$$
\frac{d}{d \sigma} \int_{m}^{1}\left(\lambda^{2}-\frac{\bar{\lambda}^{2}}{2}\right) q d q=\left(\frac{\partial \lambda}{\partial q}\right)_{q=1}-m\left(\frac{\partial \lambda}{\partial q}\right)_{q=m}-\frac{1-m^{2}}{2}\left(\frac{\partial^{2} \lambda}{\partial q^{2}}\right)_{q=\frac{m+1}{2}}
$$

Modifying functions $F(\gamma)$ and $G(\gamma)$ for a concentric channels as:

$$
\begin{aligned}
& F(\gamma)=\int_{m}^{1}\left(\lambda^{2}-\frac{\bar{\lambda}^{2}}{2}\right) q d q \\
& G(\gamma)=\left(\frac{\partial \lambda}{\partial q}\right)_{q=1}-m\left(\frac{\partial \lambda}{\partial q}\right)_{q=m}-\frac{1-m^{2}}{2}\left(\frac{\partial^{2} \lambda}{\partial q^{2}}\right)_{q=\frac{m+1}{2}}
\end{aligned}
$$

Equation (44) can be written in a similar form as:

$$
\frac{d \sigma}{d \gamma}=\frac{F^{\prime}(\gamma)}{G(\gamma)}
$$

Integratio of Eq. (46), the relation between $\sigma$ and $\gamma$ can be defined:

$$
\sigma=-\int_{\gamma}^{\infty} \frac{F^{\prime}(\tilde{\gamma})}{G(\tilde{\gamma})} d \tilde{\gamma}
$$

By assigning a value to parameter $\gamma$ and using Eq. (46), the value of dimensionless axial coordinate $(\sigma)$ can be obtained, and finally the velocity profile is known using closed form solution available in the Appendix. 


\subsection{Pressure Drop and Friction Factor}

\subsubsection{Microtube and Slit-Channel}

To evaluate the pressure drop along the channel, Eq. (8) needs to be integrated over the cross-sectional area as:

$$
-\frac{2^{2 k-5}}{k+1} \frac{\partial \tilde{p}}{\partial \sigma}=4^{k-2} \frac{d}{d \sigma} \int_{0}^{1} \lambda^{2} q^{k} d q-\left.\frac{\partial \lambda}{\partial q}\right|_{q=1}
$$

and then, integrated in the $\sigma$-direction:

$$
\Delta \tilde{p}=2(k+1) \int_{0}^{1} q^{k} \lambda^{2} d q-2+\left.2^{5-2 k}(k+1) \int_{\gamma}^{\infty} \frac{\partial \lambda}{\partial q}\right|_{q=1} \frac{F^{\prime}(\tilde{\gamma})}{G(\tilde{\gamma})} d \tilde{\gamma}
$$

One important parameter for the engineering calculations is the Fanning friction factor and the apparent friction factor. The fanning friction factor can be defined as:

$$
f \cdot R e_{D_{h}}=-2^{3-k}\left(\frac{\partial \lambda}{\partial q}\right)_{q=1},
$$

using the derived velocity profiles, the Fanning friction factor can be written as:

$$
f \cdot \operatorname{Re}_{D_{h}}=\frac{-\gamma^{2} \alpha_{1}^{n} \mathcal{G}_{2}(\gamma)}{\mathcal{H}(\gamma)}
$$

The apparent friction factor which considers frictional loss together with the effect of acceleration in the developing region can be derived as:

$$
f_{a p p} \cdot \operatorname{Re}_{D_{h}}=\frac{\Delta \tilde{p}}{4 \sigma}
$$

\subsubsection{Concentric Annular Microchannel}

Equation (31) can be integrated over the cross-sectional area of the channel as:

$$
-\frac{1}{4} \frac{\partial \tilde{p}}{\partial \sigma}\left(1-m^{2}\right)=\frac{d}{d \sigma} \int_{m}^{1} \lambda^{2} q d q+m\left(\frac{\partial \lambda}{\partial q}\right)_{q=m}-\left(\frac{\partial \lambda}{\partial q}\right)_{q=1}
$$

Integrating the above equation along the axial direction of channel results in:

$$
\Delta \tilde{p}=\frac{4}{1-m^{2}} \int_{m}^{1} q \lambda^{2} d q-2-\frac{4}{1-m^{2}} \int_{\gamma}^{\infty}\left[m\left(\frac{\partial \lambda}{\partial q}\right)_{q=m}-\left(\frac{\partial \lambda}{\partial q}\right)_{q=1}\right] \frac{F^{\prime}(\tilde{\gamma})}{G(\tilde{\gamma})} d \tilde{\gamma}
$$


The Fanning friction factor based on the inner and outer radii can be derived as:

$$
\begin{aligned}
& f^{1} \cdot \operatorname{Re}=-\frac{2}{1-m}\left(\frac{\partial \lambda}{\partial q}\right)_{q=m} \\
& f^{2} \cdot \operatorname{Re}=-\frac{2}{1-m}\left(\frac{\partial \lambda}{\partial q}\right)_{q=1}
\end{aligned}
$$

Furthermore, the apparent friction factor can be calculated as:

$$
f_{a p p} \cdot R e_{D_{h}}=\frac{\Delta \tilde{p}}{4 \sigma}
$$

where $\Delta \tilde{p}$ is given in Eq. (49).

\section{Results and Discussion}

The mathematical modeling is coded by the help of the Mathematica ${ }^{\circledR}$. One critical point in the evaluation is the improper integrals given in Eqs. (26), (47), (49) and (54). The evaluation of these integrals may be problematic as $(\sigma \rightarrow \infty)$. Although these integrals need be integrated to infinity, it is known that the flow is fully-developed after a certain $\sigma$ value. Once the flow is fully-developed, the integrals can be evaluated without any problem since the fully-developed velocity profile is known.

\subsection{Verification of the Model}

Centerline velocity which is an important parameter in determination of entrance length and boundary layers growth is calculated and compared with available data in the literature for continuum gas flow in Fig. 2 to verify the accuracy of the present model. For comparison, full solution of Navier-Stokes equation together with continuity using finite element method based simulation environment COMSOL Multiphysics $^{\circledR}$, an analytical method based on eigenvalue expansion [26] and numerical result based on finite difference method from the literature [30] are included in Fig. 2. Although not presented, COMSOL model and Liu's results [30] can predict the velocity overshoot. However, a linearization is used in the solution of Duan and Muzychka [26] and their results cannot predict the overshoot. All solutions merge to the same centerline velocity in the fully-developed limit. COMSOL and Liu's results are very close to each other, and the present study and the results by [26] are on two opposite side of the numerical curves. The discrepancy is in the entrance region. All the results are within $\pm 3 \%$ uncertainty for the calculation of the centerline velocity regardless of the linearization. A recent experimental data obtained by 


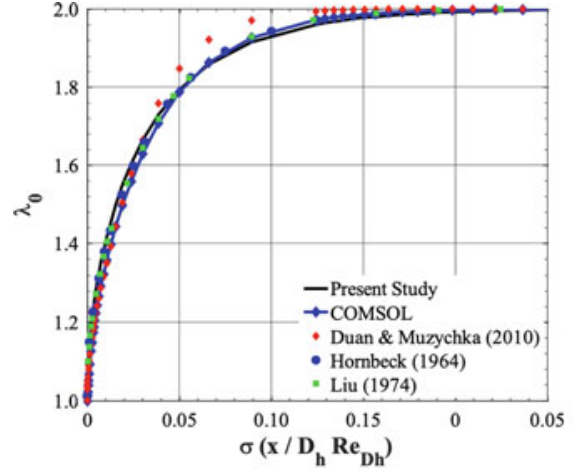

(a) Microtube

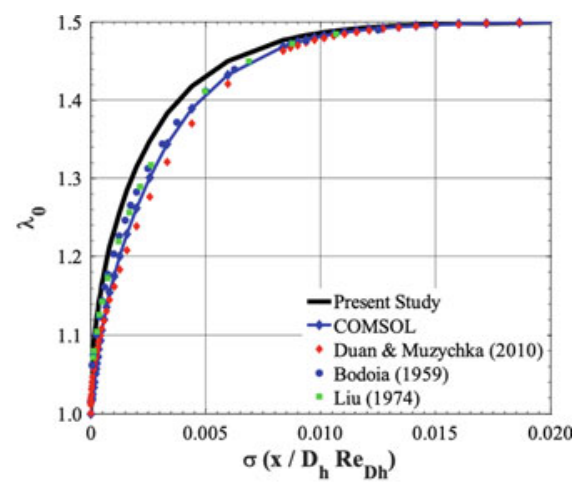

(b) Slit-channel

Fig. 2 Development of centerline velocity for continuum gas flow

Fig. 3 Comparison of velocity profile in entrance region with experimental data for a macrotube

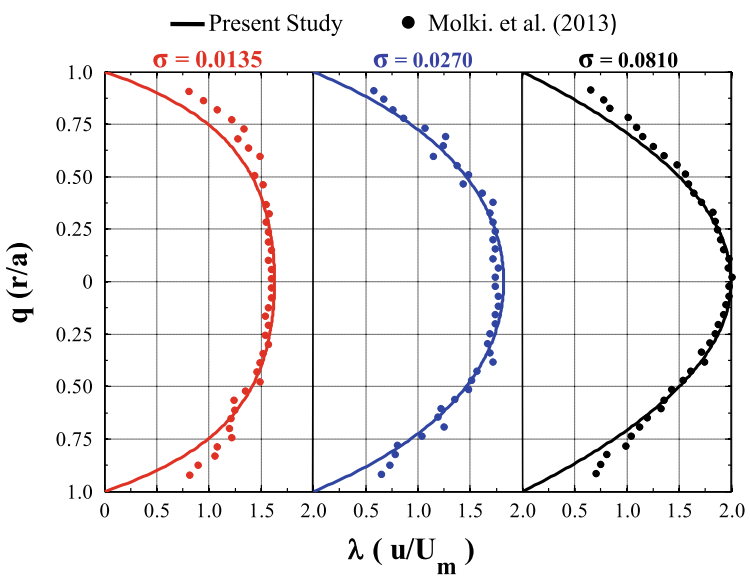

laser doppler velocimetry [29] is also compared against the result of present study for a macro-scale tube at different axial locations. The experimental data is taken at the locations above and below the centerline. As seen from the figure, the results are in good agreement. Following these verifications, it is evident that Langhaar's linearization can be implemented for modeling of velocity field within the entrance region with a reasonable accuracy (Fig. 3).

\subsection{Velocity Profile and Entrance Length}

\subsubsection{Microtube and Slit-Channel}

The developing velocity profiles within the entrance region in a microtube and slitchannel is shown in Fig. 4 for different slip models. At $\sigma=0$ which corresponds to 


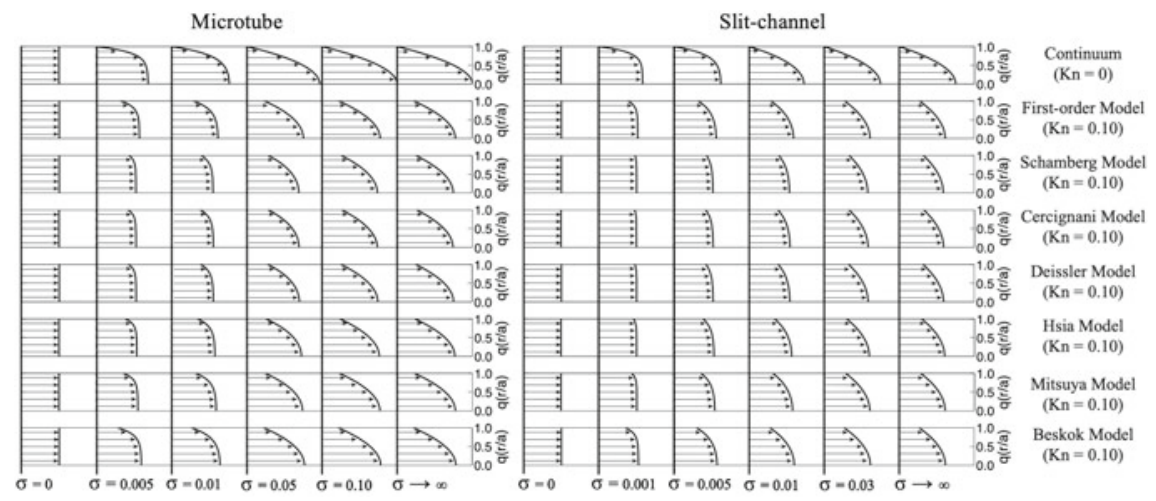

Fig. 4 Developing velocity profile within the microchannel

the inlet, a uniform velocity profile is obtained which is an inherent assumption of the boundary layer idealizations. As $\sigma$ increases, the boundary layers develop, and at a certain distance downstream, they merge and fully-developed velocity profile in the form of a parabola is achieved. In the slip-flow regime, the parabola is more flat than that of a continuum flow due to the reduction of shear stresses on the solid walls with increased $\mathcal{K} n$. Although the fully-developed profiles are similar for different models, the velocity profiles within the entrance region are quite different at some certain $\sigma$ values which leads to quite different pressure drop and friction factor characteristics. It is also worth to mention that our model cannot capture the velocity overshoot as expected. Since the sudden change of the velocity at the inlet is less pronounced for higher rarefaction, the overshoots are expected to be weak.

One parameter which is important to characterize the flow in the developing region is the entrance length, which can be defined as the required distance to downstream for which the centerline velocity reaches to $99 \%$ of the fully-developed value. The effect of slip velocity and $\mathcal{K} n$ on the entrance length is illustrated in Fig. 5 with different models. All models except Beskok model predict slower velocity development with increasing $\mathcal{K} n$. However, Beskok model predicts a reduction in entrance length after a certain $\mathcal{K} n$. This behavior can be attributed to the reduced friction on the solid walls with increased rarefaction (i.e. high $\mathcal{K} n$ ). Furthermore, the fully-developed velocity profile becomes closer to a uniform one increased rarefaction. Therefore, a shorter entrance length is quite expected.

\subsubsection{Concentric Annular Microchannel}

The velocity profiles within the entrance region of a concentric annular microchannel with a radii ratio of $m=0.5$ is given Fig. 6 for $\mathcal{K} n=0.01$. The cross-sections are chosen in the vicinity of inlet $\left(\sigma=7.4 \times 10^{-5}\right)$ and at sufficiently far downstream $\left(\sigma=1.7 \times 10^{-2}\right)$ to ensure fully-developed velocity profile. Since the velocity 


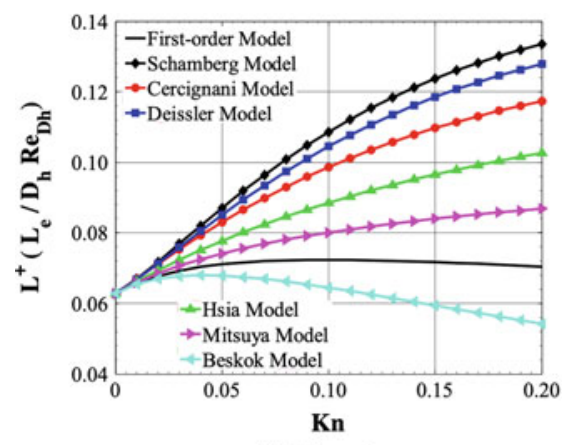

(a) Microtube

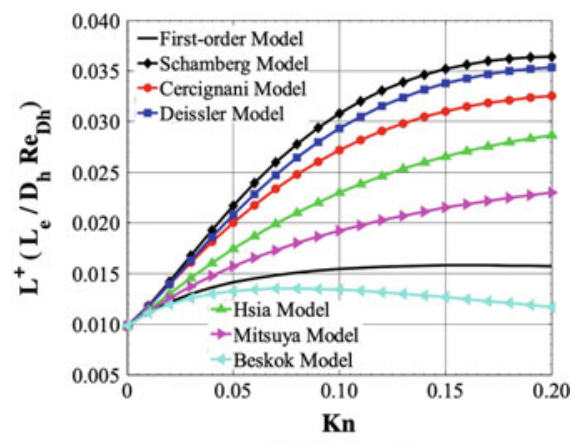

(b) Slit-channel

Fig. 5 Variation of entrance length versus $\mathcal{K} n$ for different slip models

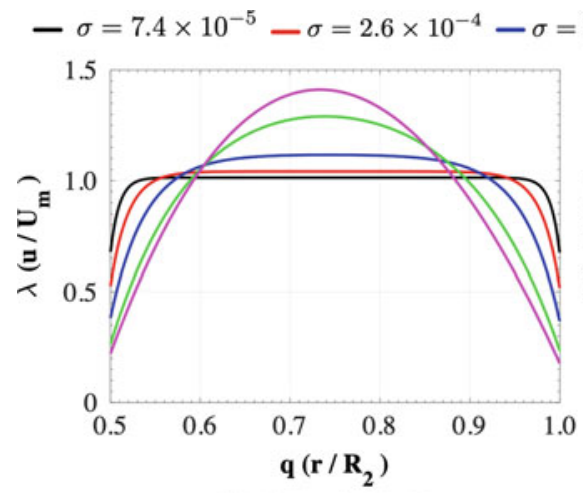

(a) First-order Model

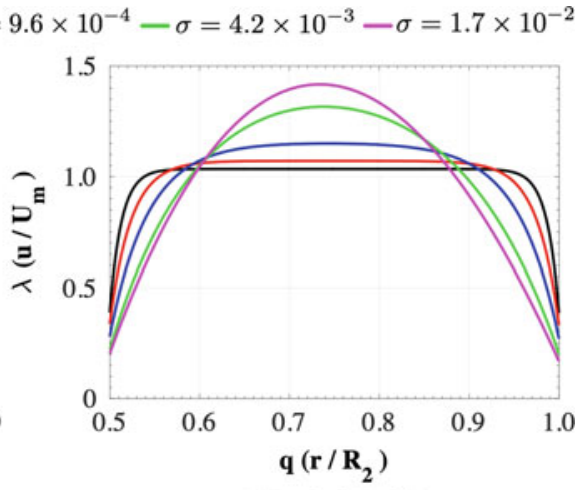

(b) Beskok Model

Fig. 6 Developing velocity profiles in a concentric annular microchannel $(\mathcal{K} n=0.01, m=0.5)$

gradients are different on the inner and outer walls which results in different slip velocities and shear stresses, the slip-velocity on the inner and outer walls are different in magnitude. Furthermore, different slip velocities on the solid walls cause non-symmetrical distribution of velocity profile around the central core. The discrepancy among two different slip models rises in the entrance region of channel and reduces drastically in the fully-developed region. The non-dimenisonal entrance length is presented for different slip models and radii ratios $m=0.1, m=0.5$ and $m=0.8$ in Fig. 7. A lower radii ratio is a case close to the microtube, and a higher radii ratio is a case closer to the slit-channel. As expected, considering the results in Fig. 5, the entrance length decreases with increasing radii ratio, and the first-order model predicts a longer entrance region. 
Fig. 7 Variation of entrance length versus $\mathcal{K} n$ using different slip models

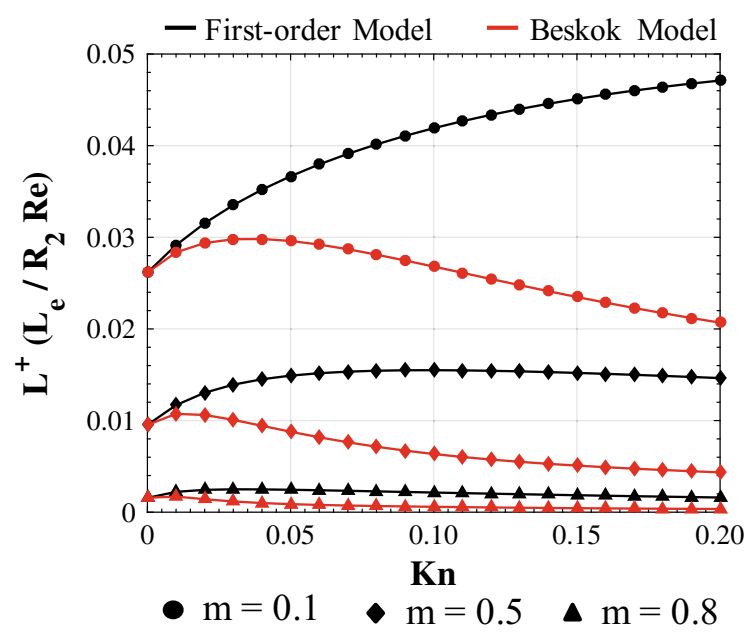

\subsection{Pressure Drop and Friction Factor}

\subsubsection{Microtube and Slit-Channel}

Pressure drop in the form of an apparent friction factor is shown in Fig. 8 both for microtube and slit-channel using different slip models and $\mathcal{K} n$. Apparent friction is also compared with available data in the literature for continuum gas flow and at the limit of the slip-flow regime. In continuum gas flow, the present model predicts apparent friction in good agreement with the data in literature. For $\mathcal{K} n=0.10$, the data of [25], which uses a first-order slip, is also included for microtube and slitchannel. For slit-channel, the result for a rectangular channel with an aspect ratio of 2.0 is included. The discrepancy between the present model and the data of [25] is due to the velocity overshoot. That is the reason, the discrepancy exists at the vicinity of the inlet, and the results converge to the same curve after a certain $\sigma$. It can be also seen that the discrepancy diminishes as $\mathcal{K} n$ increases, and the curves merge at a location closer to the inlet. The figure also shows a reduction in apparent friction with increased $\mathcal{K} n$ which can be explained by considering the reduction of shear stresses on the solid walls. The high-order models deviate from the first-order model as $\mathcal{K} n$ increases. Moreover, the asymptotic limit of the each curve is different. Again, the difference in this asymptotic value also increases with increasing $\mathcal{K} n$. Although the asymptotic values are close to each other, the apparent friction factor in the entrance region is quite different for different slip models. The apparent friction factor is more sensitive to the used slip model for shorter microchannels. 

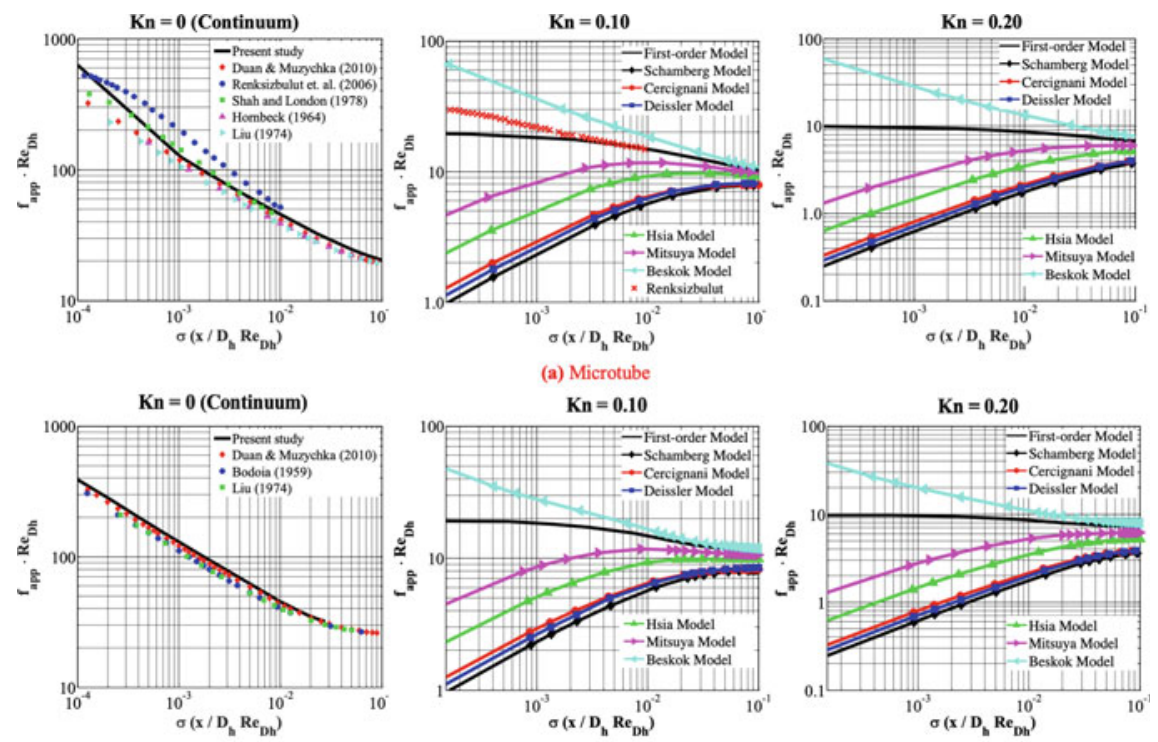

(b) Slit-channel

Fig. 8 Apparent friction factor for different $\mathcal{K} n$ and models

\subsubsection{Concentric Annular Microchannel}

Variation of apparent friction along the channel for concentric annular microchannel is illustrated in Fig. 9. Continuum regime $(\mathcal{K} n=0)$ together with two different $\mathcal{K} n$ are considered for three different radii ratios. Reduction of the apparent friction can be observed as $\mathcal{K} n$ number increases. For continuum regime flow $(\mathcal{K} n=0)$ and Beskok model, the apparent friction has a monotonic increase by approaching to inlet; however, the first-order model asymptotically reaches a constant value at the vicinity of the inlet. Far away from the inlet, all the curves reaches an asymptotic value which corresponds to the fully-developed value.
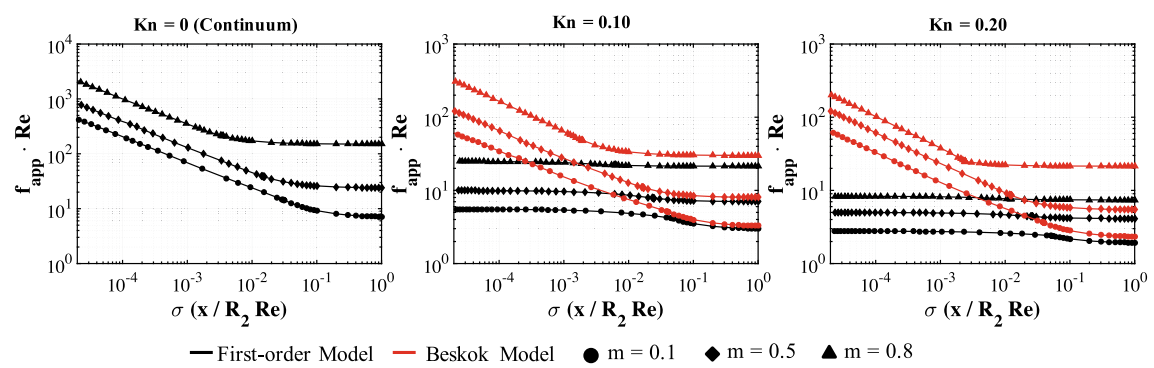

—First-order Model — Beskok Model

$\Delta \mathrm{m}=0.8$

Fig. 9 Apparent friction factor for different slip models and $\mathcal{K} n$ 


\section{Concluding Remarks}

Measurement of different flow parameters to understand fluid physics at micro-scale is still challenging. Therefore, analytical and numerical models serve as a basis for fundamental understanding of the phenomena. Present study presents an extended Langhaar's solution for the solution of a 2D incompressible, isothermal flow in a developing region of a microchannel considered both in cylindrical and cartesian coordinates. The analytical model is verified for the flows at macro-scale, and validity of the certain assumptions have been discussed. The hydrodynamic entrance length and apparent friction factor which are the quantities of interest for many engineering calculations are presented. To extend the applicability of the model, different second-order models together with the general velocity-slip boundary condition are included in the analysis. Although the applicability of the second-order models used in this study may be questionable, the present analytical model is a general mathematical tool to model the microchannel flows with high-order slip models. The model can easily be revised by the help of recent findings as long as the general form of the second-order boundary conditions is implemented. The analytical nature of the presented model enables the implementation of different slip flow models, and a fast evaluation of the velocity field which can be extended for the exploration of the combined entrance heat transfer problems for microchannels.

\section{Appendix: Velocity Profile for Concentric Annular Microchannel}

Velocity profile is expressed as

$$
\lambda(\gamma, \mathrm{q})=\mathcal{A}(\gamma) I_{0}(\gamma \mathrm{q})+\mathcal{B}(\gamma) K_{0}(\gamma \mathrm{q})+\mathcal{C}(\gamma)
$$

Using the first-order slip model coefficients $\mathcal{A}, \mathcal{B}$ and $\mathcal{C}$ can be defined as:

$$
\mathcal{A}=\frac{\mathcal{A}_{1}^{(f)}}{\mathcal{A}_{2}^{(f)}}, \quad \mathcal{B}=\frac{\mathcal{B}_{1}^{(f)}}{\mathcal{B}_{2}^{(f)}}, \quad \mathcal{C}=\frac{\mathcal{C}_{1}^{(f)}}{\mathcal{C}_{2}^{(f)}}
$$

Coefficient $\mathcal{A}$ can be expressed as:

$$
\begin{aligned}
& \mathcal{A}_{1}^{(f)}=\gamma\left(m^{2}-1\right) \Pi_{1} \\
& \mathcal{A}_{2}^{(f)}=\left[\Delta_{1} I_{1}(\gamma m)-\Delta_{2} I_{0}(\gamma m)-2 I_{1}(\gamma)\right] \Pi_{1} \\
& \quad-\left[\Delta_{1} K_{1}(\gamma m)-\Delta_{2} K_{0}(\gamma m)-2 K_{1}(\gamma)\right] \Pi_{2}
\end{aligned}
$$


where

$$
\begin{aligned}
& \Pi_{1}=2 \gamma \mathcal{K} n\left(m^{2}-1\right)\left[K_{1}(\gamma m)+K_{1}(\gamma)\right]+K_{0}(\gamma m)-K_{0}(\gamma) \\
& \Pi_{2}=2 \gamma \mathcal{K} n\left(m^{2}-1\right)\left[I_{1}(\gamma m)+I_{1}(\gamma)\right]-I_{0}(\gamma m)+I_{0}(\gamma) \\
& \Delta_{1}=2 \gamma^{2} \mathcal{K} n\left(m^{2}-1\right)+2 m, \quad \Delta_{2}=\gamma\left(m^{2}-1\right)
\end{aligned}
$$

Coefficient $\mathcal{B}$ can be expressed as:

$$
\begin{aligned}
& \mathcal{B}_{1}^{(f)}=\gamma\left(m^{2}-1\right) \Pi_{2} \\
& \mathcal{B}_{2}^{(f)}=\Pi_{3} I_{1}(\gamma m)+\Pi_{4} I_{0}(\gamma m)+\Pi_{5} I_{1}(\gamma)+\Pi_{6} I_{0}(\gamma)-4 / \gamma
\end{aligned}
$$

where

$$
\begin{aligned}
\Pi_{3} & =-\Delta_{4} K_{1}(\gamma)+\Delta_{1} K_{0}(\gamma) \\
\Pi_{4} & =\left(\Delta_{5}+2\right) K_{1}(\gamma)-\Delta_{2} K_{0}(\gamma) \\
\Pi_{5} & =\Delta_{4} K_{1}(\gamma m)+\Delta_{3} K_{0}(\gamma m) \\
\Pi_{6} & =\Delta_{1} K_{1}(\gamma m)+\Delta_{2} K_{0}(\gamma m) \\
\Delta_{3} & =2 \gamma^{2} \mathcal{K} n\left(m^{2}-1\right)+2, \quad \Delta_{4}=4 \gamma \mathcal{K} n(m+1)[\gamma \mathcal{K} n(m-1)+1], \quad \Delta_{5}=\Delta_{1}-2 m
\end{aligned}
$$

Coefficient $\mathcal{C}$ can be expressed as:

$$
\begin{aligned}
& \mathcal{C}_{1}^{(f)}=\left[\Delta_{5} I_{1}(\gamma m)-\Delta_{2} I_{0}(\gamma m)\right] \Pi_{7}-\left[\Delta_{5} I_{1}(\gamma)-\Delta_{2} I_{0}(\gamma)\right] \Pi_{8} \\
& \mathcal{C}_{2}^{(f)}=-\mathcal{B}_{2}^{(f)}
\end{aligned}
$$

where

$$
\begin{aligned}
& \Pi_{7}=2 \gamma \mathcal{K} n K_{1}(\gamma)-K_{0}(\gamma) \\
& \Pi_{8}=2 \gamma \mathcal{K} n K_{1}(\gamma m)+K_{0}(\gamma m)
\end{aligned}
$$

Using general slip model, coefficients $\mathcal{A}, \mathcal{B}$ and $\mathcal{C}$ can also be expressed as:

$$
\mathcal{A}=\frac{\mathcal{A}_{1}^{(g)}}{\mathcal{A}_{2}^{(g)}}, \quad \mathcal{B}=\frac{\mathcal{B}_{1}^{(g)}}{B_{2}^{(g)}}, \quad \mathcal{C}=\frac{\mathcal{C}_{1}^{(g)}}{\mathcal{C}_{2}^{(g)}}
$$

Coefficient $\mathcal{A}$ can be expressed as:

$$
\begin{aligned}
\mathcal{A}_{1}^{(g)}= & \left(1-m^{2}\right) \Pi_{9} \\
\mathcal{A}_{2}^{(g)}= & \left\{\Delta_{6} I_{1}(\gamma m)+\left(m^{2}-1\right) I_{0}(\gamma m)-2 / \gamma\left[m I_{1}(\gamma m)+I_{1}(\gamma)\right]\right\} \Pi_{9} \\
& +\left\{\Delta_{6} K_{1}(\gamma m)-\left(m^{2}-1\right) K_{0}(\gamma m)-2 / \gamma\left[m K_{1}(\gamma m)+K_{1}(\gamma)\right]\right\} \Pi_{10}
\end{aligned}
$$


where

$$
\begin{aligned}
\Pi_{9} & =\frac{2 \gamma \mathcal{K} n}{b_{1} \mathcal{K} n-1} K_{1}(\gamma m)-K_{0}(\gamma m)+\frac{2 \gamma \mathcal{K} n}{b_{2} \mathcal{K} n-1} K_{1}(\gamma)+K_{0}(\gamma) \\
\Pi_{10} & =-\frac{2 \gamma \mathcal{K} n}{b_{1} \mathcal{K} n-1} I_{1}(\gamma m)-I_{0}(\gamma m)-\frac{2 \gamma \mathcal{K} n}{b_{2} \mathcal{K} n-1} I_{1}(\gamma)+I_{0}(\gamma) \\
\Delta_{6} & =\frac{2 \gamma \mathcal{K} n\left(m^{2}-1\right)}{b_{1} \mathcal{K} n-1}
\end{aligned}
$$

Coefficient $\mathcal{B}$ can be expressed as:

$$
\begin{aligned}
& \mathcal{B}_{1}^{(g)}=\Delta_{5}\left[\tilde{b}_{2} I_{1}(\gamma m)+\tilde{b}_{1} I_{1}(\gamma)\right]+\Delta_{2} \tilde{b}_{1} \tilde{b}_{2}\left[I_{0}(\gamma m)+I_{0}(\gamma)\right] \\
& \mathcal{B}_{2}^{(g)}=\Pi_{11} I_{1}(\gamma m)+\Pi_{12} I_{0}(\gamma m)+\Pi_{13} I_{1}(\gamma)+\Pi_{14} I_{0}(\gamma)-4 \tilde{b}_{1} \tilde{b}_{2} / \gamma
\end{aligned}
$$

where

$$
\begin{aligned}
\Pi_{11} & =2 \gamma \mathcal{K} n\left(2 \tilde{b}_{1} m+2 \tilde{b}_{2}-\Delta_{5}\right) K_{1}(\gamma)+\tilde{b}_{2}\left(2 \tilde{b}_{1} m-\Delta_{5}\right) K_{0}(\gamma) \\
\Pi_{12} & =\tilde{b}_{1}\left(2 \tilde{b}_{2}-\Delta_{5}\right) K_{1}(\gamma)-\Delta_{2} \tilde{b}_{1} \tilde{b}_{2} K_{0}(\gamma) \\
\Pi_{13} & =-2 \gamma \mathcal{K} n\left(2 \tilde{b}_{1} m+2 \tilde{b}_{2}-\Delta_{5}\right) K_{1}(\gamma m)+\tilde{b}_{1}\left(2 \tilde{b}_{2}-\Delta_{5}\right) K_{0}(\gamma m) \\
\Pi_{14} & =\tilde{b}_{2}\left(2 \tilde{b}_{1} m-\Delta_{5}\right) K_{1}(\gamma m)+\Delta_{2} \tilde{b}_{1} \tilde{b}_{2} K_{0}(\gamma m) \\
\tilde{b}_{1} & =b_{1} \mathcal{K} n-1, \quad \tilde{b}_{2}=b_{2} \mathcal{K} n-1
\end{aligned}
$$

The coefficients $b_{1}$ and $b_{2}$ are defined in Eq. (38). Coefficient $\mathcal{C}$ can be expressed as:

$$
\begin{aligned}
& \mathcal{C}_{1}^{(g)}=\left[\Delta_{5} I_{1}(\gamma m)+\tilde{b}_{1} \Delta_{2} I_{0}(\gamma m)\right] \Pi_{15}+\left[\Delta_{5} K_{1}(\gamma m)+\tilde{b}_{1} \Delta_{2} K_{0}(\gamma m)\right] \Pi_{16} \\
& \mathcal{C}_{2}^{(g)}=-\mathcal{B}_{2}^{(g)}
\end{aligned}
$$

where

$$
\begin{gathered}
\Pi_{15}=2 \gamma \mathcal{K} n K_{1}(\gamma)+\tilde{b}_{2} K_{0}(\gamma) \\
\Pi_{16}=-2 \gamma \mathcal{K} n I_{1}(\gamma)+\tilde{b}_{2} I_{0}(\gamma)
\end{gathered}
$$

Fully-developed velocity profile using first-order slip model slip model can be written as:

$$
\lambda_{f d}^{(f)}=\frac{\mathcal{Q}_{1}}{\mathcal{Q}_{2}}
$$


where

$$
\begin{aligned}
\mathcal{Q}_{1}= & 2(m-1)\left\{8 \mathcal{K} n^{2}\left(1-m^{2}\right)+2 \mathcal{K} n\left(m^{3}+1\right)-2 \mathcal{K} n q^{2}(m+1)+\log \left[\frac{\left.q^{m(m+1)(1-m+4 K n}\right)}{m^{m\left(1-q^{2}+4 \mathscr{K} n\right)}}\right]\right\} \\
\mathcal{Q}_{2}= & \left(m^{2}-1\right)\left[8 \mathcal{K} n^{2}(m+2)+m^{2}(1-2 \mathcal{K} n)-2 \mathcal{K} n\right] \\
& +\left[(1-m)\left(m^{2}+1\right)+8 \mathcal{K} n\left(m^{2}-m+1\right)\right] \log m^{m}
\end{aligned}
$$

and for general slip model, fully-developed velocity can be written as:

$$
\lambda_{f d}^{(g)}=\frac{\mathcal{S}_{1}}{\mathcal{S}_{2}}
$$

where

$$
\begin{aligned}
\mathcal{S}_{1}=2\left(m^{2}-1\right)\{ & \log q^{m}\left[\tilde{b}_{1} \tilde{b}_{2}\left(m^{2}-1\right)+4 \mathcal{K} n\left(\tilde{b}_{1} m-\tilde{b}_{2}\right]\right. \\
& +\log m^{m} \tilde{b}_{2}\left[\tilde{b}_{1}\left(q^{2}-1\right)+4 \mathcal{K} n\right] \\
& \left.+2 \mathcal{K} n\left[\tilde{b}_{1}\left(1-q^{2}\right)+\tilde{b}_{2} m \mathcal{K} n\left(m^{2}-q^{2}\right)+4 \mathcal{K} n\left(m^{2}-1\right)-m^{3}(m+1)\right]\right\} \\
\mathcal{S}_{2}= & 8 \mathcal{K} n^{2} \log m^{m}\left[b_{1} m^{3}\left(m b_{2}+1\right)+b_{2}\left(1-b_{1}\right)\right] \\
& -\mathcal{K} n^{2}\left(m^{2}-1\right)\left[\left(m^{2}-1\right)\left(b_{1} b_{2} m+2 b_{1}-16\right)-2 b_{2} m\left(m^{2}+3\right)+4 b_{1} m^{2}\right] \\
& \times \mathcal{K} n\left[2\left(1-m^{5}\right)+8 m^{2}(m-1)-8 \log m^{m}\left(1+m^{3}\right)+6 m\left(m^{3}-1\right)\right] \\
& +\left[\log \left(m^{m^{5}-m}\right)-m\left(m^{2}-1\right)^{2}\right]\left[1-b_{1} b_{2} \mathcal{K} n\right]
\end{aligned}
$$

The coefficients $b_{1}$ and $b_{2}$ are defined in Eq. (39), and $\tilde{b}_{1}=b_{1} \mathscr{K} n-1$ and $\tilde{b}_{2}=$ $b_{2} \mathcal{K} n-1$.

\section{References}

1. Cetin, B., Ozer, M.B., Solmaz, M.E.: Microfluidic bio-particle manipulation for biotechnology. Biochem. Eng. J. 92, 63-82 (2014)

2. Guckenberger, D.J., de Groot, T.E., Wan, A.M.D., Beebe, D.J., Young, E.W.K.: Micromilling: a method for ultra-rapid prototyping of plastic microfluidic devices. Lab Chip 15, 2364-2378 (2015)

3. Sugioka, K., Jian, X., Dong, W., Hanada, Y., Wang, Z., Cheng, Y., Midorikawa, K.: Femtosecond laser 3d micromachining: a powerful tool for the fabrication of microfluidic, optofluidic, and electrofluidic devices based on glass. Lab Chip 14, 3447-3458 (2014) 
4. Kerse, C., Kalaycioglu, H., Elahi, P., Cetin, B., Kesim, D.K., Akcaalan, O., Yavas, S., Asik, M.D., Öktem, B., Hoogland, H., Holzwarth, R., Ilday, F.O.: Ablation-cooled material removal with ultrafast bursts of pulses. Nature 537, 84-88 (2016)

5. Yaman, M., Khudiyev, T., Ozgur, E., Kanik, M., Aktas, O., Ozgur, E.O., Deniz, H., Korkut, E., Bayindir, M.: Arrays of indefinitely long uniform nanowires and nanotubes. Nat. Mater. 10(7), 494-501 (2011)

6. Gad-el Hak, M.: The fluid mechanics of microdevices-the freeman scholar lecture. J. Fluids Eng. 121(1), 5-33 (1999)

7. Colin, S.: Rarefaction and compressibility effects on steady and transient gas flows in microchannels. Microfluid. Nanofluid. 1(3), 268-279 (2005)

8. Agrawal, A.: A comprehensive review on gas flow in microchannels. Int. J. Micro-Nano Scale Transp. 2(1), 1-40 (2011)

9. Karniadakis, G., Beskok, A., Aluru, N.: Microflows and Nanoflows: Fundamentals and Simulation. Springer Science \& Business Media (2006)

10. Dongari, N., Agrawal, A., Agrawal, A.: Analytical solution of gaseous slip flow in long microchannels. Int. J. Heat Mass Transf. 50(17-18), 3411-3421 (2007)

11. Zhang, W.-M., Meng, G., Wei, X.: A review on slip models for gas microflows. Microfluid. Nanofluid. 13(6), 845-882 (2012)

12. Colin, S., Lalonde, P., Caen, R.: Validation of a second-order slip flow model in rectangular microchannels. Heat Transfer Eng. 25(3), 23-30 (2004)

13. Duan, Z.: Second-order gaseous slip flow models in long circular and noncircular microchannels and nanochannels. Microfluid. Nanofluid. 12(5), 805-820 (2012)

14. Hadjiconstantinou, N.G.: Comment on cercignani's second-order slip coefficient. Phys. Fluids 15(8), 2352-2354 (2003)

15. Hadjiconstantinou, N.G.: Validation of a second-order slip model for dilute gas flows. Microscale Thermophys. Eng. 9(2), 137-153 (2005)

16. Beskok, A., Karniadakis, G.E.: Report: A model for flows in channels, pipes, and ducts at micro and nano scales. Microscale Thermophys. Eng. 3(1), 43-77 (1999)

17. Xue, H., Fan, Q.: A new analytic solution of the navier-stokes equations for microchannel flows. Microscale Thermophys. Eng. 4(2), 125-143 (2000)

18. Arkilic, E.B., Schmidt, M.A., Breuer, K.S.: Gaseous slip flow in long microchannels. J. Microelectromech. Syst. 6(2), 167-178 (1997)

19. Zohar, Y., Lee, S.Y.K., Lee, W.-Y., Jiang, L., Tong, P.: Subsonic gas flow in a straight and uniform microchannel. J. Fluid Mech. 472, 125-151 (2002)

20. Gat, A., Frankel, I., Weihs, D.: Gas flows through constricted shallow micro-channels. J. Fluid Mech. 602, 427-442 (2008)

21. Langhaar, H.L.: Steady flow in the transition length of a straight tube. J. Appl. Mech. 9(2), 55-58 (1942)

22. Sparrow, E.M., Lin, S.H., Lundgren, T.S.: Flow development in the hydrodynamic entrance region of tubes and ducts. Phys. Fluids 7(3), 338-347 (1964)

23. McComas, S.T.: Hydrodynamic entrance lengths for ducts of arbitrary cross section. J. Basic Eng. 89(4), 847-850 (1967)

24. Chen, R.-Y.: Flow in the entrance region at low reynolds numbers. J. Fluids Eng. 95(1), 153-158 (1973)

25. Renksizbulut, M., Niazmand, H., Tercan, G.: Slip-flow and heat transfer in rectangular microchannels with constant wall temperature. Int. J. Therm. Sci. 45(9), 870-881 (2006)

26. Duan, Z., Muzychka, Y.S.: Slip flow in the hydrodynamic entrance region of circular and noncircular microchannels. J. Fluids Eng. 132(1), 011201 (2010)

27. Darbandi, M., Schneider, G.E.: Numerical study of the flow behavior in the uniform velocity entry flow problem. Numer. Heat Transf. Part A Appl. 34(5), 479-494 (1998)

28. Berman, N.S., Santos, V.A.: Laminar velocity profiles in developing flows using a laser doppler technique. AIChE J. 15, 323-327 (1969)

29. Molki, A., Khezzar, L., Goharzadeh, A.: Measurement of fluid velocity development in laminar pipe flow using laser doppler velocimetry. Euro. J. Phys. 34(5), 1127 (2013) 
30. Shah, R.K., London, A.L.: Laminar Flow Forced Convection in Ducts: A Source Book for Compact Heat Exchanger Analytical Data. Academic Press, Cambridge (1978)

31. Sugino, Eitaro: Velocity distribution and pressure drop in the laminar inlet of a pipe with annular space. Bull. JSME 5(20), 651-655 (1962)

32. Han, L.S.: Hydrodynamic entrance lengths for incompressible laminar flow in rectangular ducts. J. Appl. Mech. 27(3), 403-409 (1960)

33. Harley, J.C., Huang, Y., Bau, H.H., Zemel, J.N.: Gas flow in micro-channels. J. Fluid Mech. 284, 257-274 (1995)

34. Arkilic, E.B., Breuer, K.S., Schmidt, M.A.: Mass flow and tangential momentum accommodation in silicon micromachined channels. J. Fluid Mech. 437, 29-43 (2001)

35. Cercignani, C., Lorenzani, Silvia: Variational derivation of second-order slip coefficients on the basis of the boltzmann equation for hard-sphere molecules. Phys. Fluids 22(6), 062004 (2010)

36. Cetin, B., Yazicioglu, A.G., Kakac, S.: Slip-flow heat transfer in microtubes with axial conduction and viscous dissipation-An extended Graetz problem. Int. J. Therm. Sci. 48, 1673-1678 (2009)

37. Cetin, B., Bayer, O.: Evaluation of Nusselt number for a flow in a microtube using second-order slip model. Therm. Sci. 15(Suppl. 1), 103-109 (2011)

38. Çetin, Barbaros: Effect of Thermal Creep on Heat Transfer for a Two-Dimensional Microchannel Flow: An Analytical Approach. J. Heat Transf. 135(10), 101007-101008 (2013)

39. Çetin, B., Zeinali, S.: Analysis of heat transfer and entropy generation for a low-Peclet-number microtube flow using a second-order slip model: an extended-Graetz problem. J. Eng. Math. 89(1), 13-25 (2014) 